Research Article

\title{
Competition Analysis on Industry Populations Based on a Three-Dimensional Lotka-Volterra Model
}

\author{
Sheng-Yuan Wang $\mathbb{D}^{1,2}$ Wan-Ming Chen, ${ }^{1}$ and Xiao-Lan $\mathrm{Wu}^{2}$ \\ ${ }^{1}$ College of Economics and Management, Nanjing University of Aeronautics and Astronautics, Nanjing, Jiangsu 210016, China \\ ${ }^{2}$ Nanjing XiaoZhuang University, Nanjing, Jiangsu 211171, China \\ Correspondence should be addressed to Sheng-Yuan Wang; 56439976@qq.com
}

Received 12 April 2021; Revised 23 June 2021; Accepted 12 July 2021; Published 21 July 2021

Academic Editor: A. E. Matouk

Copyright $\odot 2021$ Sheng-Yuan Wang et al. This is an open access article distributed under the Creative Commons Attribution License, which permits unrestricted use, distribution, and reproduction in any medium, provided the original work is properly cited.

\begin{abstract}
The classical Lotka-Volterra model is mainly a two-dimensional configuration. The two-dimensional form limits the application scenarios of the Lotka-Volterra model. The research objective of this paper is to develop a feasible three population equilibrium model to analyze the industry competition. In order to expand the Lotka-Volterra model to empirical analysis, this paper proposes a symbiosis model of enterprise population based on a three-dimensional Lotka-Volterra model. This paper analyzes the symbiotic system from two aspects: the balanced development of three species and the competitive evolution of the three species, taking the sales volume of three local automobile enterprises in China as the sample for empirical analysis. The symbiotic optimization under the equilibrium state of three populations indicates that there is the possibility of equilibrium and reciprocity in the growth of three automobile product populations. The empirical analysis fully explains the feasibility of this research paradigm. The analysis of competition evolution shows that cooperative action is better than competitive strategy and that comprehensive cooperation is the ideal state of a three-population symbiotic system.
\end{abstract}

\section{Introduction}

Research for competing for interacting networks takes into account real-world networks [1-6]. However, the researchers ignored the competitors competing not only to survive but also to defeat their competitors [7]. An incursion between species can alter the dynamics of the predator-prey interaction [8], which is a widespread phenomenon. The theoretical framework that measures the feedback mechanisms and resilience dynamics between two competing dynamic networks was developed to identify the factors in a network $[9,10]$. However, the competition network in the real world is more complex compared with the relationship between two species. The complex symbiotic relationship in the industrial ecosystem can be taken as an example.

The industrial ecosystem, which emerged in the 1990s, is an interdisciplinary applied discipline. Its main purpose is to explore the interaction between industry and its products and the natural environment. Traditional industrial activities should be transformed into an industrial ecosystem [11]. In this system, the consumption of energy and material is optimized, the output of waste is minimized, and the waste of one industrial process can be used as the raw material of another process. The theory of industrial ecology studies how the industrial system operates, regulates, and interacts with the biosphere and decides how to adjust the industry to make it compatible with the biosphere based on the cognition of the ecosystem [12]. The research of the industrial ecosystem is rich and diversified. Carayannis and Alexander [13] propose a dynamic- and learning-driven framework to study the process of knowledge generation and how to maximize organizational value by using game theory. Carayannis et al. [14] believe that knowledge sharing and knowledge management across organizational boundaries have provided a good analytical framework for the research cooperation between industry and university and promoted the trust relationship and social capital between organizational cooperation. Korhonen et al. [15] point out that the concept of sustainable development of 
industrial ecosystem provides a new way of thinking and an innovation path for the current industrial system construction, and the theory of industrial ecosystem and interorganizational management can supplement the shortcomings of the traditional theory of intraorganizational environmental management and guide human behavior to achieve the goal of industrial ecological balance.

Many scholars have made in-depth research on the practical application of the industrial ecosystem. Cabezas et al. [16] hold that the development of industrial ecology should not only stay at the stage of concept proposal but also be realized in practice. Therefore, simulated ecological and technological experiments have been conducted, and it is further pointed out that the industrial ecosystem is a complex sustainable system and that a complex "generalized food web" is formed in industry, agriculture, and natural organisms of human social activities. Korhonen and Snakin [17] scientifically analyze the application of industrial ecology to the ecological economy, environmental planning, and organization management by establishing various models of resource utilization, energy cycle, and industrial diversification. They emphasize that industrial ecology and industrial ecological evolution theory will play an important role in the future development of the industrial economy. In addition, Basu and van Zyl [18] further combine the theory of industrial ecology and the theory of cleaner production and point out that the degree of cleaner production depends on the degree of industrial ecology. The research findings of these scholars enrich the theoretical system of industrial ecology and provide a new research perspective for the harmonious development of the economy and environment.

The industrial ecosystem provides a systematic analysis and a synthetic method to examine the whole life cycle of a product or a process, not just the impact of a part of a certain stage. Lotka-Volterra model (LV model) is a classical model for the study of coevolution among species in natural ecology. It is proposed by Lotka [19] and Volterra [20] to analyze the competition mechanism between two species. Lotka-Volterra model is based on the logistic curve, which is an extension of the logistic model under the condition of nonsingle species competition. Based on the logistic growth curve of a single species, the dynamic competitive growth of multiple agents in the system is considered. Lotka-Volterra model was successfully spread to the research fields of the social and economic system to interpret the competition behavior between organizations [21]. The model is widely used in the research fields of industrial competition, enterprise competition, and market competition [22-25]. Some researchers use the Lotka-Volterra model to analyze innovation activities among competitors [26-29] or technology substitution among competitors [30, 31]. Some researchers focus on the transformation path [32].

At present, the application of the multidimensional Lotka-Volterra model is rare. In fact, the classical Lotka-Volterra model mainly explains the symbiotic relationship between two populations. The interpretation of symbiotic relationships in the real world needs the help of more complex models. As a result, this paper provides a three-dimensional Lotka-Volterra approach to analyze the competition evolution dynamic among the firms. Ecological populations and communities in nature are composed of organisms with different life characteristics. There are various complex organic relations between different populations, groups, and organisms and the natural environment. Under the comprehensive effect of the external environment, the ecological community develops and evolves continuously, thus forming an ecosystem. Similar to the evolution of the natural ecological community, the industrial ecological innovation system is also an open and dynamic system. There are many similarities between them in selection mechanism, environmental function, evolution, and development. The evolution process of industrial ecological innovation system can be analyzed and illustrated by referring to the evolution phenomenon among biological populations. Geroski and Mazzucato [33] explored four models for industry population dynamics: market size model, negative feedback model, contagion model of entry and exit, and density-dependent model.

At present, few researchers focus on the application of industrial ecosystem theory to a certain industry. This paper attempts to study the evolution path of specific industrial ecological innovation systems from the perspective of ecology through a combination of the theory of industrial ecosystem and specific industries and to make some tentative exploration on the evolution and development of industries. The research objective of this paper is to develop a feasible three population equilibrium model to analyze the industry competition. The benefits of the three-dimensional Lotka-Volterra system are as follows: we successfully develop a feasible three population equilibrium model to analyze the industry competition, and that expands the research situation of the two-dimensional model and has a broader application field. The three-dimensional Lotka-Volterra model is closer to the real-world population competition relationship. The two-dimensional Lotka-Volterra model can be regarded as a special case of the three-dimensional Lotka-Volterra model. It is an ideal state that there are only two competitors in an industry. This paper proves the value of the three-dimensional Lotka-Volterra model from both theoretical and empirical perspectives and successfully expands the system dimension of the Lotka-Volterra model in the analysis of the competitive relationship.

\section{Materials and Methods}

In this section, we will describe the three-dimensional Lotka-Volterra model and its equilibrium state. These theorems will be used to discuss the case of industrial competition analysis. This paper selects BYD, Great Wall Motor, and ChangAn Motor as the research objects. These three companies have the same market segment and are among the top domestic auto companies in this market segment.

As shown in Figure 1, this paper provides the deduction of the LV-MCGP model. The specific model construction process is as follows.

2.1. Dynamics Analysis of Logistic and Lotka-Volterra Models. According to the logistic model, we constructed an internal dynamic system of population $1\left(P_{1}\right)$. 


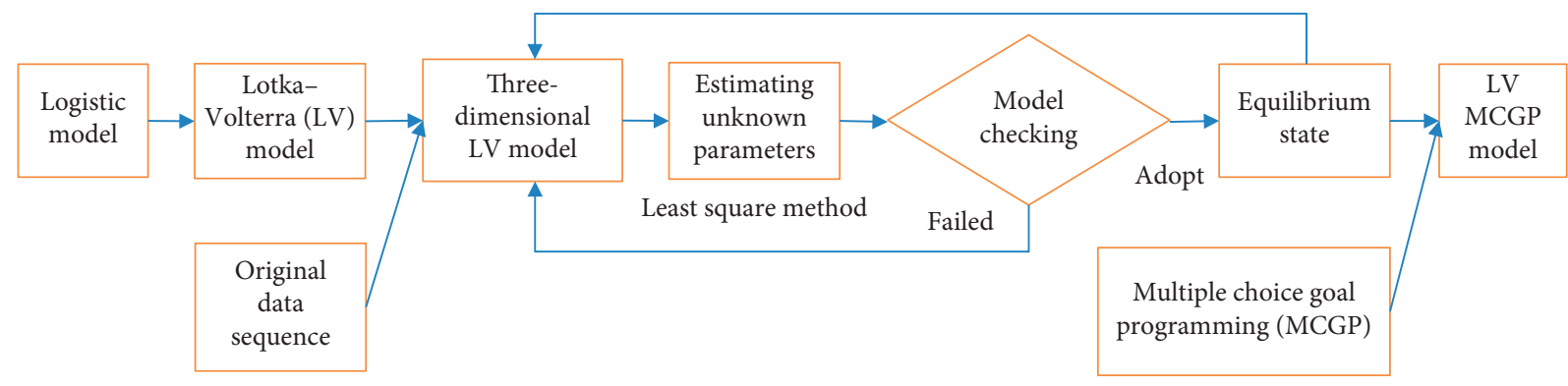

FIGURE 1: Research flow chart.

$$
g_{1}(t)=\frac{\mathrm{d} N_{1}(t)}{\mathrm{d} t}=\alpha_{1} N_{1}\left(1-\frac{N_{1}}{K_{1}}\right),
$$

where $g_{1}(t)$ is the growth rate in phase $t . N_{1}(t)$ is the population size of period $t . K_{1}$ is the maximum population scale. $\alpha_{1}$ is the intrinsic growth rate. $\left(1-\left(N_{1} / K_{1}\right)\right)$ is the retardation of growth.

The measurement model is as follows:

$$
\begin{gathered}
\text { because } N_{1}(t) \approx \Delta N_{1}(t), \Delta N_{1}(t)=N_{1}(t)-N_{1}(t-1), \mathrm{d} t \approx \Delta t=t-(t-1)=1 \\
\text { so } g_{1}(t) \approx \Delta N(t)=\gamma_{1} N_{1}(t-1)+\gamma_{2} N_{1}^{2}(t-1)
\end{gathered}
$$

Among them, $\gamma_{1}=\alpha_{1}$; usually, $\gamma_{1}>1$. It usually represents the synergy within a population. $\gamma_{2}=-\left(\alpha_{1} / K_{1}\right)$; usually, $\gamma_{2}<0$. It refers to the competition effect within a population. It is called the internal competition coefficient or population density inhibition coefficient.

Similarly, the internal relation model of population 2 $\left(P_{2}\right)$ can be obtained:

$$
g_{2}(t)=\frac{\mathrm{d} N_{2}(t)}{\mathrm{d} t}=\alpha_{2} N_{2}\left(1-\frac{N_{2}}{K_{2}}\right) .
$$

The following system shows the impact of the $P_{2}$ on $P_{1}$ :

$$
g_{1}(t)=\frac{\mathrm{d} N_{1}(t)}{\mathrm{d} t}=\alpha_{1} N_{1}\left(1-\frac{N_{1}}{K_{1}}+\frac{\beta_{12} N_{2}}{K_{2}}\right),
$$

where $\beta_{12}$ is the influence of population 2 on population 1 $\left(\beta_{12}>0\right.$, synergistic effect, $\beta_{12}<0$, competitive effect). Similarly, the following system shows the impact of $P_{1}$ on $P_{2}$ :

$$
g_{2}(t)=\frac{\mathrm{d} N_{2}(t)}{\mathrm{d} t}=\alpha_{2} N_{2}\left(1-\frac{N_{2}}{K_{2}}+\frac{\beta_{21} N_{1}}{K_{1}}\right),
$$

where $\beta_{21}$ is the impact factor of population 1 on population 2. The dynamic system of $P_{1}$ and $P_{2}$ is

$$
\left\{\begin{array}{l}
g_{1}(t)=\frac{\mathrm{d} N_{1}(t)}{\mathrm{d} t}=\alpha_{1} N_{1}\left(1-\frac{N_{1}}{K_{1}}+\frac{\beta_{12} N_{2}}{K_{2}}\right), \\
g_{2}(t)=\frac{\mathrm{d} N_{2}(t)}{\mathrm{d} t}=\alpha_{2} N_{2}\left(1-\frac{N_{2}}{K_{2}}+\frac{\beta_{21} N_{1}}{K_{1}}\right) .
\end{array}\right.
$$

Equation (6) is called the Lotka-Volterra (LV) system. The LV model, based on the logistic model of a single species, considers the dynamic growth of competition and symbiosis of two or more entities simultaneously in the ecosystem [20], which can accurately describe the competition and symbiosis between corporate populations. The LV system can determine the influence of the core population in the evolution of the entire ecosystem [34] and thus has better data fitting and prediction expression [35].

The classical Lotka-Volterra model, a differential dynamic system, was used to simulate the dynamic relationship of populations in ecology. Later, economists introduced it into the fluctuation of macroeconomic growth and the market competition of medium scale and scope. According to the principle of biology, there are many kinds of functional relationships among biological populations: promotion or inhibition. For their own survival and development, there is also a relationship between market competition subjects: the existence of one subject can promote or inhibit the diffusion process of another. The LV model of dualpopulation or multipopulation growth is a differential dynamic system to simulate the dynamic relationship between populations. Based on the numerical value of $\beta$, the type of interaction between species can be judged [36]:

(1) When $\beta_{12}=0$ and $\beta_{21}=0$, the populations develop independently and do not affect each other. At this time, the LV model expresses no symbiotic relationship.

(2) When $\beta_{12}<0$ and $\beta_{21}<0$, the two populations compete with each other. One party grows while the other declines. There is no symbiotic relationship between the two.

(3) When $\beta_{12}>0$ and $\beta_{21}<0$ or $\beta_{21}<0$ and $\beta_{21}>0$, one population is attached to the other during the symbiotic evolution of the innovative population, showing a parasitic mode of constantly requesting 
resources from the other population to maintain its own growth.

(4) When $\beta_{12}>0$ and $\beta_{21}=0$ or $\beta_{12}=0$ and $\beta_{21}>0$, both sides have obtained extra high-quality resources in the evolution process, but the symbiosis coefficient of one of them is zero, indicating that it has not obtained extra resources, suffering from no loss, and the innovation ecosystem is now in a symbiotic mode of partial benefit.

(5) When $\beta_{12}>0$ and $\beta_{21}>0$, the population is in a mutually beneficial symbiosis mode. Among them, if $\beta_{12} \neq \beta_{21}$, the symbiotic relationship between the two populations is asymmetric and mutually beneficial symbiosis; when $\beta_{12}=\beta_{21}$, the populations have obtained equal benefits in the process of symbiotic evolution, and the resources are exchanged in equal amounts, forming a symmetric and mutually beneficial symbiosis.

In business activities, competition can occur between populations that use common resources. Symbiosis in the industry ecosystem does not exclude competition. Populations in completely or partially the same living space need to conduct technology, talent, and market interaction in the factor market. However, when one population in the ecosystem relies on another core or dominant population to obtain resources and living space, a parasitic relationship is formed. In a parasitic relationship, the symbiotic subject has a one-way exchange of interests. The state is not extensive because of this asymmetric one-way exchange. Therefore, the system will gradually develop in the direction of symbiosis conducive to mutual dependence and mutual benefit.

2.2. Analysis of Equilibrium Point Stability. The equilibrium point of the evolution of the populations means that the output of both parties has reached the maximum and has remained stable. The following does the stability analysis. In a stable state, the equations are

$$
\left\{\begin{array}{l}
f_{1}\left(N_{1}, N_{2}\right) \equiv \frac{\mathrm{d} N_{1}(t)}{\mathrm{d} t}=\alpha_{1} N_{1}\left(1-\frac{N_{1}}{K_{1}}+\frac{\beta_{12} N_{2}}{K_{2}}\right)=0 \\
f_{2}\left(N_{1}, N_{2}\right) \equiv \frac{\mathrm{d} N_{2}(t)}{\mathrm{d} t}=\alpha_{2} N_{2}\left(1-\frac{N_{2}}{K_{2}}+\frac{\beta_{21} N_{1}}{K_{1}}\right)=0 .
\end{array}\right.
$$

The equilibrium points are $P_{1}(0,0), P_{2}\left(N_{1}, 0\right), P_{3}(0$, $\left.N_{2}\right), \quad P_{4}\left(\left(K_{1}\left(1+\beta_{12}\right) /\left(1-\beta_{12} \beta_{21}\right)\right), \quad\left(K_{2}\left(1+\beta_{21}\right) /\left(1-\beta_{12}\right.\right.\right.$ $\left.\left.\beta_{21}\right)\right)$ ).

The Jacobian matrix is used to solve the equilibrium point. We obtain the determinant Det $(J)$ and trace $\operatorname{Tr}(J)$ of the Jacobian matrix. When Det $(J)>0$ and $\operatorname{Tr}(J)<0$, the local equilibrium point is in a stable state; otherwise, it is not a stable equilibrium point. Table 1 shows a comparative analysis of the determinants and traces of the Jacobian matrix. On this basis, stable equilibrium points are obtained.

As shown in Table 1, the condition in which the local equilibrium point is stable is obtained. Through comparison, the symbiosis evolution mode under different values corresponds to different stable equilibrium points, but the equilibrium points of partial benefit, asymmetric reciprocity, and symmetric reciprocity symbiosis are all the same $P_{4}$. The parasitic equilibrium point may be $P_{2}, P_{3}$, or $P_{4}$ (Table 2).

As shown in Table 2, the symbiosis evolution equilibrium point of the population is related to the symbiosis model and is affected by the size of the mutual competition coefficient between the innovation populations and the maximum capacity of the respective populations.

Because of the interdependence between the two populations, the size of the population should not be zero. $P_{4}$ point corresponds to the scale of population $1\left(P_{1}\right)$ and population $2 \quad\left(P_{2}\right), \quad$ respectively $\left(\left(K_{1}\left(1+\beta_{12}\right)\right) /\left(1-\beta_{12} \beta_{21}\right)\right),\left(\left(K_{2}\left(1+\beta_{21}\right)\right) /\left(1-\beta_{12} \beta_{21}\right)\right)$. At the same time, the conditions for meaningful $P_{4}$ are

$$
\left\{\begin{array}{l}
\frac{K_{1}\left(1+\beta_{12}\right)}{1-\beta_{12} \beta_{21}}>0 \\
\frac{K_{2}\left(1+\beta_{21}\right)}{1-\beta_{12} \beta_{21}}>0
\end{array}\right.
$$

With the solution of the above equations, the only nonnegative solution can be obtained. That is, the equilibrium solution is $\left(\left(K_{1}\left(1+\beta_{12}\right)\right) /\left(1-\beta_{12} \beta_{21}\right)\right)$, $\left(\left(K_{2}\left(1+\beta_{21}\right)\right) /\left(1-\beta_{12} \beta_{21}\right)\right)$. This equilibrium solution represents the equilibrium state of the LV system.

2.3. Three-Dimensional Lotka-Volterra Model. In a symbiotic system composed of population $1\left(P_{1}\right)$, population 2 $\left(P_{2}\right)$, and population $3\left(P_{3}\right)$, the mathematical model is

$$
\left\{\begin{array}{l}
g_{1}(t)=\frac{\mathrm{d} N_{1}(t)}{\mathrm{d} t}=\alpha_{1} N_{1}\left(1-\frac{N_{1}}{K_{1}}+\frac{\beta_{12} N_{2}}{K_{2}}+\frac{\beta_{13} N_{3}}{K_{3}}\right) \\
g_{2}(t)=\frac{\mathrm{d} N_{2}(t)}{\mathrm{d} t}=\alpha_{2} N_{2}\left(1-\frac{N_{2}}{K_{2}}+\frac{\beta_{21} N_{1}}{K_{1}}+\frac{\beta_{23} N_{3}}{K_{3}}\right) \\
g_{3}(t)=\frac{\mathrm{d} N_{3}(t)}{\mathrm{d} t}=\alpha_{3} N_{3}\left(1-\frac{N_{3}}{K_{3}}+\frac{\beta_{31} N_{1}}{K_{1}}+\frac{\beta_{32} N_{2}}{K_{2}}\right) .
\end{array}\right.
$$

Among them, $\beta_{i j}(i=1,2,3, j=1,2,3$. $)$ is the interaction coefficient between populations. When $\beta_{i j}$ is greater than zero, there is a synergetic relationship among populations. When $\beta_{i j}$ is less than zero, there is competition among populations.

Objective programming is an effective way to solve the problem of multiobjective programming. The basic idea is to determine the expected value of each objective function of the multiobjective programming problem. The goal programming method is more flexible, more effective, and easier to use and implement in dealing with multiobjective problems. In recent years, multichoice goal programming (MCGP) has been widely used to solve many practical decision problems [37]. Chang [38] proposes the multiplechoice goal programming (MCGP) method as 


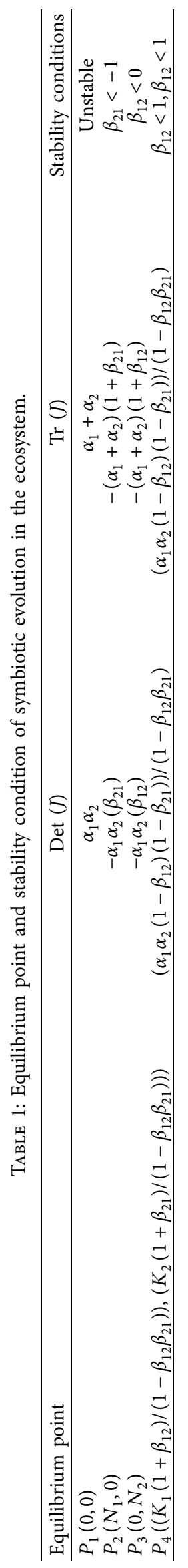


TABLE 2: Symbiotic evolution model and equilibrium point of the ecosystem.

\begin{tabular}{lcc}
\hline Value combination of influencing factor & Symbiotic evolution model among populations & Stable equilibrium point \\
\hline$\beta_{12} \beta_{21}<0$ & Parasitic relationship & $P_{2}, P_{3}, P_{4}$ \\
$\beta_{12}=0, \beta_{21}<0$. or $\beta_{21}=0, \beta_{12}<0$ & Favor symbiosis & $P_{4}$ \\
$\beta_{12}>0, \beta_{21}>0, \beta_{12} \neq \beta_{21}$ & Asymmetric symbiosis & $P_{4}$ \\
$\beta_{12}>0, \beta_{21}>0, \beta_{12}=\beta_{21}$ & Symmetry symbiosis & $P_{4}$ \\
\hline
\end{tabular}

$$
c\left\{\begin{array}{l}
\text { objective function, } \\
\operatorname{Min} \sum_{i=1}^{n}\left(d_{i}^{+}+d_{i}^{-}\right)+\sum_{i=1}^{n}\left(e_{i}^{+}+e_{i}^{-}\right), \\
\text {constraints, } \\
g_{i}=f_{i}(x)-d_{i}^{+}+d_{i}^{-}, \quad i=1,2, \ldots, n, \\
x \in X=\left\{x_{1}, x_{2}, \ldots, x_{m}\right\}, \\
X \in F,(F \text { is the set of feasible solutions }), \\
g_{i, \max }=g_{i}-e_{i}^{+}+e_{i}^{-}, \quad i=1,2, \ldots, n, \\
g_{i, \max } \geq g_{i} \geq g_{i, \min }, \quad i=1,2, \ldots, n, \\
e_{i}^{+}, e_{i}^{-}, d_{i}^{+}, d_{i}^{-} \geq 0, \quad i=1,2, \ldots, n .
\end{array}\right.
$$

Here, $d_{i}^{+}$and $d_{i}^{-}$indicate the value of the $i$-th goal exceeding and not reaching the expected value of the goal. $f_{i}(x)$ is the formula of the $i$-th objective. $X$ is the decision variable. $g_{i}$ indicates the expected goal of $i$-th. $e_{i}^{+}$are close to the positive deviation of $\left|g_{i}-g_{i, \max }\right| . e_{i}^{-}$are close to the negative deviation of $\left|g_{i}-g_{i, \max }\right| . g_{i, \min }$ and $g_{i, \max }$ are the lower and upper limits for the goal of $g_{i}$. Having studied the relevant research [39], the authors embed the equilibrium condition of the LV system into the MCGP model to obtain the Lotka-Volterra-MCGP model in this paper:

objective function, $\operatorname{Min} \sum_{i=1}^{n}\left(d_{i}^{+}+d_{i}^{-}\right)+\sum_{i=1}^{n}\left(e_{i}^{+}+e_{i}^{-}\right)$,

constraints,

$g_{i}=f_{i}(x)+d_{i}^{+}-d_{i}^{-}, \quad i=1,2, \ldots, n$,

$x \in X, \quad X=\left\{x_{1}, x_{2}, \ldots, x_{m}\right\}$,

$X \in F$ ( $F$ is the set of feasible solutions),

$g_{i, \max }=g_{i}+e_{i}^{-}-e_{i}^{+}, \quad i=1,2, \ldots, n$,

$g_{i, \min } \leq g_{i}, g_{i} \leq g_{i, \max }, \quad i=1,2, \ldots, n$,

$d_{i}^{+}, d_{i}^{-}, e_{i}^{+}, e_{i}^{-} \geq 0, \quad i=1,2, \ldots, n$,

$g_{1}(t)=\frac{\mathrm{d} N_{1}(t)}{\mathrm{d} t}=\alpha_{1} N_{1}\left(1-\frac{N_{1}}{K_{1}}+\frac{\beta_{12} N_{2}}{K_{2}}+\frac{\beta_{13} N_{3}}{K_{3}}\right)$,

$g_{2}(t)=\frac{\mathrm{d} N_{2}(t)}{\mathrm{d} t}=\alpha_{2} N_{2}\left(1-\frac{N_{2}}{K_{2}}+\frac{\beta_{21} N_{1}}{K_{1}}+\frac{\beta_{23} N_{3}}{K_{3}}\right)$,

$g_{3}(t)=\frac{\mathrm{d} N_{3}(t)}{\mathrm{d} t}=\alpha_{3} N_{3}\left(1-\frac{N_{3}}{K_{3}}+\frac{\beta_{31} N_{1}}{K_{1}}+\frac{\beta_{32} N_{2}}{K_{2}}\right)$,

$-1<\beta_{i j}<1, \quad i=1,2,3, j=1,2,3$,

$\sum g_{i}=K$, ( $K$ is the sum of market capacity).
As a linear form for goal programming, multichoice goal programming embedded with Lotka-Volterra equilibrium (LV-MCGP) can be easily resolved by common software.

\section{Results and Discussion}

This paper takes competitive enterprises in the automobile industry as an example. The automobile industry is one of the largest and most important industries in the world. In a sense, the development level and strength of the automobile industry reflect the comprehensive national strength and competitiveness of a country. China's automobile production and sales have ranked first in the world for many years in a row, and India, Russia, and other countries have also developed rapidly in recent years. The emerging market is characterized by low car ownership per capita and large potential demand, so it is still the most potential market for the world automobile industry in the future. China Industry Information Network (CIIN) shows that auto production increased from 2.07 million to 24.5 million during 2000-2015, with an average annual growth rate of $17.92 \%$ [40]. After years of rapid development, the growth state of China's vehicle industry has returned stable [41].

In the medium and long term, the impact of national policy-oriented market consumption demand is relatively short-lived, and the automobile industry has a huge pulling effect on the economy because it has a high contribution to GDP growth. Under the premise of stable growth, it is difficult to introduce policies to stimulate or inhibit the automobile industry. The influence of local purchase restriction policy on the overall sales of the automobile industry is limited, so the sustainable development of the social economy is the decisive factor for the sustainable growth of the automobile industry.

This study selects the independent brands in China's automobile market (providing average quality and low-end products) as the market segment for analysis. Three major manufacturers in the market segment are taken as the research samples. The products of three enterprises are regarded as three product groups. The scale of product population is expressed by the sales volume of automobile products in each quarter. The three-dimensional LV system is used to study the symbiotic and interaction mechanism among three enterprises (three enterprise product populations). The status of the automobile industry in China's national economy is constantly improving, but it is also facing increasingly fierce and cruel global competition. Given the coexistence of opportunities and challenges, it has become a realistic proposition how to comprehensively, correctly, and timely evaluate the competitive situation of 
automobile manufacturing enterprises, so as to achieve the purpose of promoting the sustainable and healthy development of the automobile industry. The development level of the automobile industry depends on the level of automobile manufacturing to a certain extent. With the characteristics of a long industrial chain, high relevance, strong driving force, and integration of various high and new technologies, automobile manufacturing enterprises have become one of the important symbols to measure the industrial level, economic strength, and the ability in technological innovation of a country.

Founded in 1995, BYD is listed on Shenzhen Stock Exchange and Hong Kong stock exchange. It is mainly engaged in the business of rechargeable batteries and the automotive industry including traditional fuel vehicles and new energy vehicles.

Great Wall Motor Co., Ltd., is the largest SUV manufacturing enterprise in China. It was listed in Hong Kong H-share. At present, it has three brands of Haval, Great Wall, and Wey, and its products cover three categories of
SUV, car, and pickup truck. It has four vehicle production bases and has the independent supporting ability of engine, transmission, and other core parts.

ChangAn automobile is a camp enterprise of four major groups of Chinese automobiles. It has 159 years of history and 37 years of vehicle manufacturing accumulation.

3.1. Three-Species System Analysis. Automobile sales have significant seasonal characteristics, so this paper gets the smoothing data firstly. In this paper, the four-period simple moving average method is used to process the observation data, and the later research is mainly based on the moving average data. Auto sales data comes from the public database of related enterprise websites.

As shown in Table 3, the data provided the official website of the China Automobile Industry Association [42]. By substituting the seasonal smoothing data in Table 3 into the following econometric model and making regression analysis, Table 4 can be obtained.

$$
\left\{\begin{array}{l}
g_{1}(t)=\gamma_{11} N_{1}(t-1)+\gamma_{12} N_{1}^{2}(t-1)+\gamma_{13} N_{1}(t-1) N_{2}(t-1)+\gamma_{14} N_{1}(t-1) N_{3}(t-1), \\
g_{2}(t)=\gamma_{21} N_{2}(t-1)+\gamma_{22} N_{2}^{2}(t-1)+\gamma_{23} N_{2}(t-1) N_{1}(t-1)+\gamma_{24} N_{2}(t-1) N_{3}(t-1), \\
g_{3}(t)=\gamma_{31} N_{3}(t-1)+\gamma_{32} N_{3}^{2}(t-1)+\gamma_{33} N_{3}(t-1) N_{1}(t-1)+\gamma_{34} N_{3}(t-1) N_{2}(t-1) .
\end{array}\right.
$$

Among them, $\gamma_{1}=\alpha_{1}$. Generally, $\gamma_{1}>0$, meaning the synergy effect within the population. $\gamma_{2}=-\left(\alpha_{1} / K_{1}\right)$, and generally $\gamma_{2}<0$, meaning the competition effect within the population. It is called the internal competition coefficient or the population density inhibition coefficient.

The results of the regression are shown in Table 4.

As shown in Table 4, the regression effect is not very good, the $P$ value is high, and the correlation coefficient value does not meet the requirements of the theoretical hypothesis.

Among them, $\alpha_{1}=-0.103, K_{1}=107015 . \alpha_{2}=-0.220$, $K_{2}=206861 . \alpha_{3}=0.773$, and $K_{3}=185888$.
In order to deal with the above problem, a step-by-step measurement method is provided to reduce the dimension of the measurement model.

Step 1. Calculate $\alpha_{i}$ and $K_{i}$ based on the population growth model.

As shown in Table 5, the effect of regression is better, which meets the requirements of the theoretical hypothesis. Substitute the above parameters into equation (12).

Step 2. Deform the model (12) as model (13). In the transformed equations, the left side of the equal sign is the known value, and the right side of the equal sign is the parameter equation waiting to be solved.

$$
\left\{\begin{array}{l}
g_{1}(t)-\gamma_{11} N_{1}(t-1)-\gamma_{12} N_{1}^{2}(t-1)=\gamma_{13} N_{1}(t-1) N_{2}(t-1)+\gamma_{14} N_{1}(t-1) N_{3}(t-1), \\
g_{2}(t)-\gamma_{21} N_{2}(t-1)-\gamma_{22} N_{2}^{2}(t-1)=\gamma_{23} N_{1}(t-1) N_{2}(t-1)+\gamma_{24} N_{2}(t-1) N_{3}(t-1), \\
g_{3}(t)-\gamma_{31} N_{3}(t-1)-\gamma_{32} N_{3}^{2}(t-1)=\gamma_{33} N_{1}(t-1) N_{3}(t-1)+\gamma_{34} N_{2}(t-1) N_{3}(t-1) .
\end{array}\right.
$$


TABle 3: Sample data and seasonal smoothing data of car sales.

\begin{tabular}{|c|c|c|c|c|c|c|c|}
\hline \multirow{2}{*}{ Year } & \multirow{2}{*}{ Quarter } & \multicolumn{3}{|c|}{ Sample data } & \multicolumn{3}{|c|}{ Seasonal smoothing data } \\
\hline & & BYD & Great Wall & ChangAn & BYD & Great Wall & ChangAn \\
\hline \multirow{3}{*}{2020} & 3 & 98819 & 182765 & 225284 & 92732 & 173252 & 184340 \\
\hline & 2 & 91360 & 156011 & 181133 & 93678 & 169556 & 163993 \\
\hline & 1 & 59659 & 106427 & 121559 & 96856 & 173549 & 148508 \\
\hline \multirow{4}{*}{2019} & 4 & 121090 & 247806 & 209384 & 110452 & 198452 & 161753 \\
\hline & 3 & 102602 & 167978 & 143895 & 119314 & 211231 & 144593 \\
\hline & 2 & 104071 & 171985 & 119194 & 124315 & 204440 & 145866 \\
\hline & 1 & 114044 & 206038 & 174539 & 125858 & 197640 & 152508 \\
\hline \multirow{4}{*}{2018} & 4 & 156538 & 298924 & 140742 & 125007 & 191261 & 165956 \\
\hline & 3 & 122607 & 140812 & 148988 & 118849 & 183444 & 194455 \\
\hline & 2 & 110244 & 144786 & 145761 & 110795 & 195656 & 202495 \\
\hline & 1 & 110639 & 180523 & 228332 & 106187 & 202382 & 197748 \\
\hline \multirow{4}{*}{2017} & 4 & 131904 & 267655 & 254739 & 101197 & 212539 & 203302 \\
\hline & 3 & 90391 & 189660 & 181146 & 108212 & 232547 & 195767 \\
\hline & 2 & 91812 & 171689 & 126775 & 115218 & 238035 & 194257 \\
\hline & 1 & 90681 & 221152 & 250547 & 119637 & 241703 & 197278 \\
\hline \multirow{4}{*}{2016} & 4 & 159963 & 347688 & 224598 & 122254 & 234505 & 190065 \\
\hline & 3 & 118414 & 211612 & 175106 & 116883 & 200851 & 182063 \\
\hline & 2 & 109491 & 186361 & 138860 & 105920 & 182496 & 177858 \\
\hline & 1 & 101149 & 192358 & 221696 & 104079 & 173141 & 189035 \\
\hline \multirow{4}{*}{2015} & 4 & 138477 & 213071 & 192588 & 110110 & 165619 & 181415 \\
\hline & 3 & 74562 & 138192 & 158287 & 110433 & 151124 & 166541 \\
\hline & 2 & 102126 & 148941 & 183568 & 114427 & 142207 & 152951 \\
\hline & 1 & 125273 & 162271 & 191218 & 114969 & 127553 & 132417 \\
\hline \multirow{4}{*}{2014} & 4 & 139769 & 155092 & 133092 & 109431 & 107331 & 108318 \\
\hline & 3 & 90540 & 102524 & 103924 & 108174 & 88897 & 94418 \\
\hline & 2 & 104295 & 90325 & 101433 & 113155 & 82093 & 82161 \\
\hline & 1 & 103121 & 81384 & 94823 & 116615 & 75343 & 71124 \\
\hline \multirow{4}{*}{2013} & 4 & 134741 & 81355 & 77491 & 126547 & 69930 & 64589 \\
\hline & 3 & 110464 & 75308 & 54898 & - & - & - \\
\hline & 2 & 118133 & 63325 & 57283 & - & - & - \\
\hline & 1 & 142851 & 59733 & 68684 & - & - & - \\
\hline
\end{tabular}

Unit: vehicle.

TABLE 4: Regression results of the three-species Lotka-Volterra model.

\begin{tabular}{ccccc}
\hline$g_{i}(t)$ & $\gamma_{i 1}$ & $\gamma_{i 2}$ & $\gamma_{i 3}$ & $\gamma_{i 4}$ \\
\hline$g_{1}(t)$ & $-0.103(P=0.505)$ & $2.823 * 10^{-7}(P=0.820)$ & $-1.094 * 10^{-6}(P=0.011)$ & $1.581 * 10^{-6}(P=0.004)$ \\
$g_{2}(t)$ & $-0.220(P=0.280)$ & $-1.851 * 10^{-6}(P=0.002)$ & $3.788 * 10^{-6}(P=0.025)$ & $9.563 * 10^{-7}(P=0.175)$ \\
$g_{3}(t)$ & $0.773(P \leq 0.001)$ & $-2.314 * 10^{-6}(P=0.003)$ & $-4.046 * 10^{-6}(P=0.019)$ & $5.386 * 10^{-7}(P=0.326)$ \\
\hline
\end{tabular}

Let $\alpha_{i}$ and $K_{i}$ be substituted into the model. The value of $Y_{i}$ can be obtained by calculating the following equations:

$$
\left\{\begin{array}{l}
Y_{1}=g_{1}(t)-\gamma_{11} N_{1}(t-1)-\gamma_{12} N_{1}^{2}(t-1) \\
Y_{2}=g_{2}(t)-\gamma_{21} N_{2}(t-1)-\gamma_{22} N_{2}^{2}(t-1) \\
Y_{3}=g_{3}(t)-\gamma_{31} N_{3}(t-1)-\gamma_{32} N_{3}^{2}(t-1)
\end{array}\right.
$$

Equation (14) gives the value of $Y_{i}$. The model can then continue to calculate the variables of the relationship between populations. A new econometric model (15) can be obtained.

$$
\left\{\begin{array}{l}
Y_{1}=\gamma_{13} N_{1}(t-1) N_{2}(t-1)+\gamma_{14} N_{1}(t-1) N_{3}(t-1) \\
Y_{2}=\gamma_{23} N_{1}(t-1) N_{2}(t-1)+\gamma_{24} N_{2}(t-1) N_{3}(t-1) \\
Y_{3}=\gamma_{33} N_{1}(t-1) N_{3}(t-1)+\gamma_{34} N_{2}(t-1) N_{3}(t-1)
\end{array}\right.
$$

Step 3. Get the relevant parameters through regression analysis. The research replaces $\gamma_{i j}$ in the equations with $\beta_{i j}$ and $K_{i}$, and then the value of $\beta_{i j}$ can be obtained by regression operation. 
TABLE 5: Calculation results of $\alpha_{i}$ and $K_{i}$ values.

\begin{tabular}{lr}
\hline Variable & Regression coefficient \\
\hline$\alpha_{1}$ & $0.181(P=0.109)$ \\
$-\left(\alpha_{1} / K_{1}\right)$ & $-1.697 * 10^{-6}(P=0.107)$ \\
$K_{1}$ & 107015 \\
$\alpha_{2}$ & $0.231(P=0.002)$ \\
$-\left(\alpha_{2} / K_{2}\right)$ & $-1.119 * 10^{-6}(P=0.003)$ \\
$K_{2}$ & 206861 \\
$\alpha_{3}$ & $0.301(P=0.001)$ \\
$-\left(\alpha_{2} / K_{2}\right)$ & $-1.621 * 10^{-6}(P=0.001)$ \\
$K_{3}$ & 185888 \\
\hline
\end{tabular}

As shown in Table 6, the regression results are provided. The regression coefficients of some parameters are not significant. This shows that there is a difference between the symbiosis model and the ideal state in the real world.
Step 4. Put the regression results into the three-species symbiosis system. The results are as follows:

$$
\left\{\begin{array}{l}
g_{1}(t)=0.181 N_{1}(t-1)-\frac{0.181}{107015} N_{1}^{2}(t-1)+\frac{0.181 \times(-1.069)}{206861} N_{1}(t-1) N_{2}(t-1)+\frac{0.181 \times 0.944}{185888} N_{1}(t-1) N_{3}(t-1) \\
g_{2}(t)=0.231 N_{2}(t-1)-\frac{0.231}{206861} N_{2}^{2}(t-1) \\
g_{3}(t)=0.301 N_{3}(t-1)-\frac{0.301}{185888} N_{3}^{2}(t-1)
\end{array}\right.
$$

By observing the above formula, we can find that this is a typical asymmetric three-species symbiosis model. Product group 2 (Great Wall Motor) has a competitive (negative) impact on product group 1 (BYD), while product group 3 (ChangAn Motor) has a cooperative (positive) impact on product group 1 (BYD). In order to understand the interaction mechanism of this symbiotic system, we will analyze the evolution from the perspectives of three-species equilibrium development and three-species dynamic game.

3.2. Balanced Development of Symbiotic System Analysis. The three-species equilibrium system can be obtained by modifying the actual system (formula (16)).

$$
\Rightarrow\left\{\begin{array}{l}
g_{1}(t)=0.181 N_{1}-\frac{0.181}{107015} N_{1}^{2}+\frac{0.181 \times \beta_{12}}{206861} N_{1} N_{2}+\frac{0.181 \times \beta_{13}}{185888} N_{1} N_{3}, \\
g_{2}(t)=0.231 N_{2}-\frac{0.231}{206861} N_{2}^{2}+\frac{0.231 \times \beta_{21}}{107015} N_{2} N_{1}+\frac{0.231 \times \beta_{23}}{185888} N_{2} N_{3} \\
g_{3}(t)=0.301 N_{3}-\frac{0.301}{185888} N_{3}^{2}+\frac{0.301 \times \beta_{31}}{107015} N_{3} N_{1}+\frac{0.301 \times \beta_{32}}{206861} N_{3} N_{2} .
\end{array}\right.
$$

The equilibrium point of the three-species symbiosis system is as follows:

$$
\left\{\begin{array}{l}
g_{1}(t)=0.181 N_{1}-\frac{0.181}{107015} N_{1}^{2}+\frac{0.181 \times \beta_{12}}{206861} N_{1} N_{2}+\frac{0.181 \times \beta_{13}}{185888} N_{1} N_{3}=0 \\
g_{2}(t)=0.231 N_{2}-\frac{0.231}{206861} N_{2}^{2}+\frac{0.231 \times \beta_{21}}{107015} N_{2} N_{1}+\frac{0.231 \times \beta_{23}}{185888} N_{2} N_{3}=0 \\
g_{3}(t)=0.301 N_{3}-\frac{0.301}{185888} N_{3}^{2}+\frac{0.301 \times \beta_{31}}{107015} N_{3} N_{1}+\frac{0.301 \times \beta_{32}}{206861} N_{3} N_{2}=0
\end{array}\right.
$$


TABLE 6: Regression results of interaction coefficient.

\begin{tabular}{lccr}
\hline Interaction coefficient & Regression value & $P$ value & Significance \\
\hline$\beta_{12}$ & -1.069 & $(0.019)$ & Significant \\
$\beta_{13}$ & 0.944 & $(0.037)$ & Significant \\
$\beta_{21}$ & 0.199 & $(0.523)$ & Not significant \\
$\beta_{23}$ & -0.358 & $(0.307)$ & Not significant \\
$\beta_{31}$ & 0.022 & $(0.940)$ & Not significant \\
$\beta_{32}$ & -0.011 & $(0.972)$ & Not significant \\
\hline
\end{tabular}

The equivalent change can be obtained as follows:

$$
\Rightarrow\left\{\begin{array}{l}
N_{1}-\frac{1}{107015} N_{1}^{2}+\frac{\beta_{12}}{206861} N_{1} N_{2}+\frac{\beta_{13}}{185888} N_{1} N_{3}=0, \\
N_{2}-\frac{1}{206861} N_{2}^{2}+\frac{\beta_{21}}{107015} N_{2} N_{1}+\frac{\beta_{23}}{185888} N_{2} N_{3}=0, \\
N_{3}-\frac{1}{185888} N_{3}^{2}+\frac{\beta_{31}}{107015} N_{3} N_{1}+\frac{\beta_{32}}{206861} N_{3} N_{2}=0 .
\end{array}\right.
$$

Divide both sides of the equation by $N_{i}(i=1,2,3)$.

$$
\Rightarrow\left\{\begin{array}{l}
1-\frac{1}{107015} N_{1}+\frac{\beta_{12}}{206861} N_{2}+\frac{\beta_{13}}{185888} N_{3}=0, \\
1-\frac{1}{206861} N_{2}+\frac{\beta_{21}}{107015} N_{1}+\frac{\beta_{23}}{185888} N_{3}=0, \\
1-\frac{1}{185888} N_{3}+\frac{\beta_{31}}{107015} N_{1}+\frac{\beta_{32}}{206861} N_{2}=0 .
\end{array}\right.
$$

The following formula can be obtained by equivalent transformation of the equations:

$$
\Rightarrow\left\{\begin{array}{l}
N_{1}=107015+\frac{107015}{206861} \beta_{12} N_{2}+\frac{107015}{185888} \beta_{13} N_{3} \\
=107015+0.517 \beta_{12} N_{2}+0.575 \beta_{13} N_{3}, \\
N_{2}=206861+\frac{206861}{107015} \beta_{21} N_{1}+\frac{206861}{185888} \beta_{23} N_{3} \\
=206861+1.933 \beta_{21} N_{1}+1.112 \beta_{23} N_{3}, \\
N_{3}=185888+\frac{185888}{107015} \beta_{31} N_{1}+\frac{185888}{206861} \beta_{32} N_{2} \\
=185888+1.737 \beta_{31} N_{1}+0.898 \beta_{32} N_{2} .
\end{array}\right.
$$

By substituting the above symbiotic relationship into the MCGP model, the following results are obtained: objective function, $\operatorname{Min} \sum_{i=1}^{n}\left(d_{i}^{+}+d_{i}^{-}\right)+\sum_{i=1}^{n}\left(e_{i}^{+}+e_{i}^{-}\right)$,

constraints,

$g_{i}=f_{i}(x)+d_{i}^{-}-d_{i}^{+}, \quad i=1,2, \ldots, n$,

$x \in X, \quad X=\left\{x_{1}, x_{2}, \ldots, x_{m}\right\}$,

$X \in F$, ( $F$ is the set of feasible solutions),

$g_{i, \max }=g_{i}+e_{i}^{-}-e_{i}^{+}, \quad i=1,2, \ldots, n$,

$g_{i, \min } \leq g_{i}, g_{i} \leq g_{i, \max }, \quad i=1,2, \ldots, n$,

$e_{i}^{+}, e_{i}^{-}, d_{i}^{+}, d_{i}^{-} \geq 0, \quad i=1,2, \ldots, n$,

$N_{1}=107015+\frac{107015}{206861} \beta_{12} N_{2}+\frac{107015}{185888} \beta_{13} N_{3}=107015$

$+0.517 \beta_{12} N_{2}+0.575 \beta_{13} N_{3}$,

$N_{2}=206861+\frac{206861}{107015} \beta_{21} N_{1}+\frac{206861}{185888} \beta_{23} N_{3}$

$=206861+1.933 \beta_{21} N_{1}+1.112 \beta_{23} N_{3}$,

$N_{3}=185888+\frac{185888}{107015} \beta_{31} N_{1}+\frac{185888}{206861} \beta_{32} N_{2}$

$=185888+1.737 \beta_{31} N_{1}+0.898 \beta_{32} N_{2}$,

$-1<\beta_{i j}<1, \quad i=1,2,3, j=1,2,3$,

$\sum g_{i}=K, \quad(K$ is the sum of market capacity).

The problem is solved using the LINGO [43] software. The results of $\beta_{i j}$ value optimization of the equilibrium symbiosis model are shown in Table 7 .

As shown in Table 7, with the expansion of the market scale, more cooperative behaviors among populations are required. Cooperation behavior is comprehensive, and the intensity of cooperation is similar, not the greater the better. The intensity of cooperation between enterprises is close to equilibrium. 
TABLE 7: Simulation of symbiotic relationship of balanced growth of three populations.

\begin{tabular}{lccccccc}
\hline Market size $(K)$ & 499764 & 550000 & 600000 & 650000 & 700000 & 750000 & 800000 \\
\hline$\beta_{12}$ & 0.000 & 0.000 & 0.000 & 0.000 & 0.181 & 0.181 & 0.186 \\
$\beta_{13}$ & 0.000 & 0.000 & 0.000 & 0.000 & 0.181 & 0.181 & 0.186 \\
$\beta_{21}$ & 0.000 & 0.000 & 0.181 & 0.181 & 0.171 & 0.181 \\
$\beta_{23}$ & 0.000 & 0.067 & 0.302 & 0.113 & 0.000 & 0.104 & 0.188 \\
$\beta_{31}$ & 0.000 & 0.181 & 0.000 & 0.181 & 0.181 & 0.181 & 0.188 \\
$\beta_{32}$ & 0.000 & 0.000 & 0.000 & 0.181 & 0.181 & 0.181 \\
\hline
\end{tabular}

3.3. Three-Species Evolutionary and Strategic Analysis. In order to illustrate the evolution trend of the three-species game, this paper selects several representative symbiotic relationships for data simulation:

(1) Comprehensive cooperation: the three enterprises adopt the strategic behavior of mutual cooperation $(\beta=0.5)$

(2) Overall competition: the three enterprises take the strategic behavior of competing with each other $(\beta=-0.5)$

(3) The combination of the two powers: the interaction coefficient between them is 0.5. At the same time, they take the competitive strategy against the third enterprise, and the interaction coefficient is -0.5 . In this study, Great Wall Motor and ChangAn Motor are the relative advantage enterprises. For example, in 2020, the total sales of the Great Wall and ChangAn Automobile ranked 7th and 8th in the Chinese market. BYD, meanwhile, ranked 16th in sales [42]

(4) The combination of the two weak enterprises: the interaction coefficient between them is 0.5 . At the same time, they take the competitive strategy against the third enterprise, and the interaction coefficient is -0.5 . In this study, Great Wall Motor and BYD motor are relatively inferior enterprises

(5) Isolated mode: the three enterprises have no influence on each other and develop separately

(6) Parasitic mode: one enterprise gets help from two other enterprises. BYD is an example to illustrate the parasitic mode

(7) Malicious parasitism mode: one enterprise gets help from two other enterprises and takes competitive activities against them. BYD is an example to illustrate the malicious parasitism model

The statistical characteristics of data simulation results are shown in Table 8 .

As shown in Table 8, under different models, the simulation data are significantly different. In this paper, the simulation results will be compared and evaluated.

3.4. Model Evaluation. This paper evaluates the population growth of different modes based on the similarity between ideal solution and simulation data. The higher the similarity, the better the growth. The evaluation matrix is $A$.

$$
A=\left[a_{i j}\right]_{27 \times 3} .
$$

This study determines the weight by entropy method for the three populations in the evaluation. A technique for order preference by similarity to an ideal solution (TOPSIS) was used to evaluate the similarity. The ideal scale ( $\max$ scale) of the three populations can be regarded as three criteria for evaluating the three-species Lotka-Volterra system.

Entropy weight is an objective method [44]. It is a popular method and always combined with other methods, for example, entropy combined with fuzzy VIKOR [45], entropy combined with GIS and AHP [46], and entropy combined with subjective weight [47].

The entropy weight method is provided as follows [48]. Suppose that $m$ is alternatives $\left(A_{1}, A_{2}, \ldots, A_{m}\right)$ and $n$ is criteria $\left(C_{1}, C_{2}, \ldots, C_{n}\right)$ for a decision problem. Then initial decision matrix is

$$
A=\left[\begin{array}{cccc}
a_{11} & a_{12} & \cdots & a_{1 n} \\
a_{21} & a_{22} & \cdots & a_{2 n} \\
\vdots & \vdots & \ddots & \vdots \\
a_{m 1} & a_{m 2} & \cdots & a_{m n}
\end{array}\right]=\left[a_{i j}\right]_{m \times n}
$$

Step 1: normalize the evaluation matrix.

$$
r_{i j}=\frac{a_{i j}}{\sqrt{\sum_{i=1}^{m} a_{i j}^{2}}} .
$$

Step 2: compute entropy.

$$
e_{j}=-\frac{1}{\ln m} \sum_{i=1}^{m} r_{i j} \ln r_{i j}, \quad j=1,2, \ldots, n .
$$

Step 3: calculate weights.

$$
w_{j}=\frac{1-e_{j}}{\sum_{i=1}^{n}\left(1-e_{j}\right)}, \quad j=1,2, \ldots, n .
$$

Calculated based on the data in the example, $w_{1}=0.346$, $w_{2}=0.325$, and $w_{3}=0.329$.

Hwang and Yoon [49] proposed the popular method TOPSIS to determine the best alternative. TOPSIS method is presented as follows:

Step 1: build the normalized matrix $R$. 
TABLE 8: Statistical characteristics of population growth.

\begin{tabular}{|c|c|c|c|c|c|c|c|c|c|}
\hline & \multicolumn{3}{|c|}{ Moving average of observations } & \multicolumn{3}{|c|}{ Comprehensive cooperation } & \multicolumn{3}{|c|}{ Overall competition } \\
\hline & BYD & Great Wall & ChangAn & BYD & Great Wall & ChangAn & BYD & Great Wall & ChangAn \\
\hline Average & -1252 & 3827 & 4435 & 15913 & 87556 & 99109 & -18402 & -6162 & -1425 \\
\hline Min & -13596 & -24903 & -28499 & 3670 & 28181 & 33222 & -27006 & -26034 & -20039 \\
\hline Max & 10963 & 33654 & 24099 & 21587 & 136006 & 132616 & -9392 & 10289 & 14733 \\
\hline \multirow[t]{3}{*}{ Std. } & 5905 & 12998 & 12482 & 4730 & 30826 & 29681 & 5284 & 10990 & 9779 \\
\hline & \multicolumn{3}{|c|}{ Combination of two powers } & \multicolumn{3}{|c|}{ Combination of two weak firms } & \multicolumn{3}{|c|}{ Isolated mode } \\
\hline & BYD & Great wall & ChangAn & BYD & Great wall & ChangAn & BYD & Great wall & ChangAn \\
\hline Average & -18402 & 18031 & 24399 & -1368 & 63363 & -1425 & -1245 & 40697 & 48842 \\
\hline Min & -27006 & 6366 & 13589 & -4288 & 17184 & -20039 & -4181 & 17404 & 21408 \\
\hline Max & -9392 & 25217 & 32455 & 2015 & 100071 & 14733 & 2113 & 55833 & 61194 \\
\hline \multirow[t]{3}{*}{ Std. } & 5284 & 5132 & 4818 & 1522 & & 9779 & 1655 & 10586 & 11001 \\
\hline & & Parasitic moc & & \multicolumn{3}{|c|}{ Malicious parasitism mode } & & & \\
\hline & BYD & Great wall & ChangAn & BYD & Great wall & ChangAn & & & \\
\hline Average & 15913 & 40697 & 48842 & 15913 & 5935 & 11487 & & & \\
\hline Min & 3670 & 17404 & 21408 & 3670 & -9270 & -3225 & & & \\
\hline Max & 21587 & 55833 & 61194 & 21587 & 17031 & 23357 & & & \\
\hline Std. & 4820 & 10788 & 11210 & 4820 & 7864 & 7080 & & & \\
\hline
\end{tabular}

$$
r_{i j}=\frac{a_{i j}}{\sqrt{\sum_{i=1}^{m} a_{i j}^{2}}}
$$

Step 2: build weighted normalized matrix $V$.

$$
v_{i j}=w_{j} r_{i j}, \sum_{j=1}^{n} w_{j}=1, \quad w_{j} \text { is the weight of } j \text { th criterion. }
$$

Step 3: calculate $A^{+}$and $A^{-}$.

$A^{+}$and $A^{-}$are defined in the following way:

$$
\begin{aligned}
A^{+} & =\left\{\left(\max v_{i j} \mid j \in J\right) \operatorname{or}\left(\min v_{i j} \mid j \in J^{\prime}\right)\right\}, \quad i=1,2, \ldots, m \\
& =\left\{v_{1}^{+}, v_{2}^{+}, \ldots, v_{n}^{+}\right\}, \\
A^{-} & =\left\{\left(\min v_{i j} \mid j \in J\right) \operatorname{or}\left(\max v_{i j} \mid j \in J^{\prime}\right)\right\}, \quad i=1,2, \ldots, m \\
& =\left\{v_{1}^{-}, v_{2}^{-}, \ldots, v_{n}^{-}\right\} .
\end{aligned}
$$

The positive-ideal solution (PIS) and negative-ideal solution (NIS) are determined, where $J$ and $J$ are sets of benefit and cost criteria, respectively.

Step 4: calculate the distances of each alternative from positive-ideal solution (PIS) and negative-ideal solution (NIS).

$$
\begin{aligned}
& S_{i}^{+}=\sqrt{\sum_{j=1}^{n}\left(v_{i j}-v_{j}^{+}\right)^{2}}, \quad i=1,2, \ldots, m, \\
& S_{i}^{-}=\sqrt{\sum_{j=1}^{n}\left(v_{i j}-v_{j}^{-}\right)^{2}}, \quad i=1,2, \ldots, m .
\end{aligned}
$$

Step 5: rank the order of alternatives.

$$
C_{i}^{+}=\frac{S_{i}^{-}}{S_{i}^{+}+S_{i}^{-}}, \quad 0<C_{i}^{+}<1, i=1,2, \ldots, m
$$

Here, $C_{i}^{+} \in[0,1]$ with $i=1,2, \ldots, m$. Therefore, the best alternative should be found with the order of $C_{i}^{+}$. The more value of $C_{i}^{+}$, the better. If $C_{i}^{+}$is close to 1 , the alternative $A_{i}$ is closer to the PIS.

As shown in Table 9, this paper can successfully get the evaluation ranking of populations in different modes. For the three-species symbiosis system, the comprehensive cooperation mode is the best symbiosis mode, and the comprehensive competition mode is the worst symbiosis mode. In the comprehensive cooperation mode, the three enterprises can occupy more market share. The three enterprises have a certain advantage in the market segmentation, and their cooperation can strengthen the core competitiveness. The three enterprises can strengthen their market position by establishing strategic alliances, product development cooperation, and even price collusion.

The simple parasitic mode is better than the malicious parasitic mode. The two-weak-joint mode is better than the two-strong-joint mode. The simple parasitic mode is better than the two-weak-joint mode and the two-strong-joint mode. Cooperative behavior is always better than competitive behavior. The more cooperative behavior, the better. And the less competitive behavior, the better.

3.5. Discussion. The result shows that there is a typical asymmetric three-species symbiosis system. Product group 2 (Great Wall Motor) has a competitive (negative) impact on product group 1 (BYD), while product group 3 (ChangAn Motor) has a cooperative (positive) impact on product group 1 (BYD). For the three-species symbiosis system, the comprehensive cooperation mode is the best symbiosis mode, and the comprehensive competition mode is the worst symbiosis mode. The simple parasitic mode is better than the malicious parasitic mode. Cooperative behavior is always better than competitive behavior.

The classical Lotka-Volterra model is used to discuss the symbiosis of two species. With the extensive development of related research, the two-species model is difficult to solve 
TABLE 9: Result of TOPSIS.

\begin{tabular}{|c|c|c|c|c|c|c|c|c|}
\hline Mode & $\beta_{12}$ & $\beta_{13}$ & $\beta_{21}$ & $\beta_{23}$ & $\beta_{31}$ & $\beta_{32}$ & $C_{i}^{+}$ & Rank \\
\hline Moving average of observations & -1.069 & 0.944 & 0 & 0 & 0 & 0 & 0.229 & 7 \\
\hline Comprehensive cooperation & 0.5 & 0.5 & 0.5 & 0.5 & 0.5 & 0.5 & 0.714 & 1 \\
\hline Overall competition & -0.5 & -0.5 & -0.5 & -0.5 & -0.5 & -0.5 & 0.160 & 8 \\
\hline Combination of two powers & -0.5 & -0.5 & -0.5 & 0.5 & -0.5 & 0.5 & 0.297 & 5 \\
\hline Combination of two weak & 0.5 & -0.5 & 0.5 & -0.5 & -0.5 & -0.5 & 0.389 & 4 \\
\hline Isolated mode & 0 & 0 & 0 & 0 & 0 & 0 & 0.451 & 3 \\
\hline Parasitic mode & 0.5 & 0.5 & 0 & 0 & 0 & 0 & 0.469 & 2 \\
\hline Malicious parasitism mode & 0.5 & 0.5 & -0.5 & 0 & -0.5 & 0 & 0.281 & 6 \\
\hline
\end{tabular}

practical problems. Scholars began to pay more attention to the three-species LV model. Dong et al. deal with bistable solutions for a three-species competition model with nonlocal anisotropic dispersal [50]. They demonstrate the asymptotic behavior of traveling waves and have constructed a class of front-like entire solutions. Liu et al. have found the spreading properties of a three-species competition-diffusion system, which is noncooperative [51]. That is an important theoretical result for the three-species competition system in unbounded domains. The above researchers are the forerunner of the three-species Lotka-Volterra model. The current research mainly focuses on the mathematical analysis, feasible solution, and its conditions of the three-species Lotka-Volterra model. The research of the three-species Lotka-Volterra model for empirical and applied analysis has just got off the ground. This paper provides a feasible analysis paradigm for LV symbiosis analysis of three populations, which is a meaningful attempt.

The evolution of the industrial innovation ecosystem is a complex process. The model, method, and data studied in this paper are simple and have some shortcomings in the following aspects. Firstly, the evolution of the industrial innovation ecosystem is complex in organizational evolution, science and technology, economic development, innovation culture, innovation policy, and so on. A more in-depth research process needs to consider the influence of the above complex factors. Researchers need to summarize the evolution characteristics of the industrial innovation ecosystem, such as creativity, diversity, dependence, environmental selectivity, and self-organization. This study does not explore the life cycle characteristics of industry and enterprise development. According to the life cycle theory of ecosystem, the follow-up research needs to deeply explore the evolution process of the industrial innovation ecosystem, determine four evolution stages: germination stage, growth stage, stable stage, and decline stage, and analyze the main characteristics of each stage. In the process of the formation, operation, and evolution of the industrial ecosystem, we will encounter many problems about risk, including risk identification, risk management, and risk control. In future research, researchers should strengthen the management of the operation and evolution process of the industrial innovation ecosystem, reduce the risk, and strictly control the incidence of system risk. We can quantify the operation process and evolution process of the system and establish a scoring and management system, which will help to control the system risk. In terms of case study, we should appropriately select industries other than the automobile industry to study the industrial ecosystem, such as the telecommunications industry, clothing industry, and food industry. According to the characteristics of different industries, we should explore the formation and development rules of different industrial ecosystems and expand the research field.

\section{Conclusion}

The three-dimensional Lotka-Volterra model has been successfully built with the sales data of three local automobile enterprises in China. Based on Three-Species System Analysis, it can be found that there is a symbiotic relationship among automobile enterprises and that the threespecies model can be adopted in analyzing the competition and cooperation among enterprises. Through the balanced development of Symbiotic System Analysis, the result of symbiotic optimization under the equilibrium state of three populations is obtained. Equilibrium is possible in the growth of three populations. Three-Species Evolutionary Analysis shows that cooperative action is better than competitive strategy and that this method is more practical in the analysis of enterprise competitive strategy. The findings reveal that this approach is feasible and effective in analyzing competition, evolution, and balanced development of enterprise population. It is of great importance to study the relationships among enterprises as it is helpful for enterprises to make policies of strategy. Future studies could analyze the life cycle of competition among enterprises with the three-species Lotka-Volterra model.

\section{Data Availability}

The experimental data used to support the findings of this study are included within the article.

\section{Conflicts of Interest}

The authors declare that they have no conflicts of interest.

\section{Acknowledgments}

This work was supported by the National Social Science Foundation of China (No. 20BGL203).

\section{References}

[1] U. Bastolla, M. A. Fortuna, A. Pascual-García, A. Ferrera, B. Luque, and J. Bascompte, "The architecture of mutualistic 
networks minimizes competition and increases biodiversity," Nature, vol. 458, no. 7241, pp. 1018-1020, 2009.

[2] R. P. Rohr, S. Saavedra, and J. Bascompte, "On the structural stability of mutualistic systems," Science, vol. 345, no. 6195, p. 1253497, 2014.

[3] J. Aguirre, D. Papo, and J. M. Buldú, "Successful strategies for competing networks," Nature Physics, vol. 9, no. 4, pp. 230-234, 2013.

[4] R. M. D’Souza, "Complex network: a winning strategy," Nature Physics, vol. 9, no. 4, pp. 212-213, 2013.

[5] M. Kivela, A. Arenas, M. Barthelemy, J. P. Gleeson, Y. Moreno, and M. A. Porter, "Multilayer networks," Journal of Complex Networks, vol. 2, no. 3, pp. 203-271, 2014.

[6] I. Scholtes, N. Wider, R. Pfitzner, A. Garas, C. J. Tessone, and F. Schweitzer, "Causality-driven slow-down and speed-up of diffusion in non-Markovian temporal networks," Nature Communications, vol. 5, no. 1, p. 5024, 2014.

[7] E. Thebault and C. Fontaine, "Stability of ecological communities and the architecture of mutualistic and trophic networks," Science, vol. 329, no. 5993, pp. 853-856, 2010.

[8] M. Scheffer, S. Carpenter, J. A. Foley, C. Folke, and B. Walker, "Catastrophic shifts in ecosystems," Nature, vol. 413, no. 6856, pp. 591-596, 2001.

[9] R. M. May, "Networks and webs in ecosystems and financial systems," Philosophical Transactions. Series A, Mathematical, Physical, and Engineering Sciences, vol. 371, no. 1987, Article ID 20120376, 2013.

[10] B. Podobnik, D. Horvatic, T. Lipic, M. Perc, J. M. Buldú, and H. E. Stanley, "The cost of attack in competing networks," Journal of the Royal Society Interface, vol. 12, no. 112, p. 20150770, 2015.

[11] D. Frosch and N. Gallopoulos, "Strategies for manufacturing," Scientific American, vol. 261, no. 3, pp. 94-102, 1989.

[12] S. Erkman, "Industrial ecology: an historical view," Cleaner Production, vol. 5, no. 2, pp. 23-25, 1997.

[13] E. G. Carayannis and J. Alexander, "Winning by co-opeting in strategic government-university-industry R\&D partnerships: the power of complex, dynamic knowledge networks," Journal of Technology Transfer, vol. 24, no. 3, pp. 197-210, 1999.

[14] E. G. Carayannis, J. Alexander and A. Ioannidis, Leveraging knowledge, learning, and innovation in forming strategic government-university-industry (GUI) R\&D partnerships in the US, Germany, and France," Technovation, vol. 20, no. 9, pp. 477-488, 2000.

[15] J. Korhonen, F. Von Malmborg, P. A. Strachan, and J. R. Ehrenfeld, "Management and policy aspects of industrial ecology: an emerging research agenda," Business Strategy and the Environment, vol. 13, no. 5, pp. 289-305, 2004.

[16] H. Cabezas and C. W. Pawlowski, A. L. Mayer, N. T. Hoagland, Simulated experiments with complex sustainable systems: ecology and technology: ecology and technology," Resources, Conservation and Recycling, vol. 44, no. 3, pp. 279-291, 2005.

[17] J. Korhonen and J.-P. Snäkin, "Analysing the evolution of industrial ecosystems: concepts and application," Ecological Economics, vol. 52, no. 2, pp. 169-186, 2005.

[18] A. J. Basu and D. J. A. van Zyl, "Industrial ecology framework for achieving cleaner production in the mining and minerals industry," Journal of Cleaner Production, vol. 14, no. 314, pp. 299-304, 2006.

[19] A. J. Lotka, Elements of Physical Biology, Baltimore, Williams \& Wilkins Co., Philadelphia, PA, USA, 1925.
[20] V. Volterra, "Fluctuations in the abundance of a species considered mathematically," Nature, vol. 118, no. 2972, pp. 558-560, 1926.

[21] G. Gandolfo, "Giuseppe palomba and the Lotka-volterra equations," Rendiconti Lincei, vol. 19, no. 4, pp. 347-357, 2008.

[22] V. B. Kreng and H. T. Wang, "The competition and equilibrium analysis of LCD TV and PDP TV," Technological Forecasting and Social Change, vol. 78, no. 3, pp. 448-457, 2011.

[23] S.-Y. Chiang, "An application of Lotka-Volterra model to Taiwan's transition from $200 \mathrm{~mm}$ to $300 \mathrm{~mm}$ silicon wafers," Technological Forecasting and Social Change, vol. 79, no. 2, pp. 383-392, 2012.

[24] P. Jiang, X. Yan, and L. Wang, "A viral product diffusion model to forecast the market performance of products," Discrete Dynamics in Nature and Society, vol. 2017, Article ID 9121032, 10 pages, 2017.

[25] H.-T. Wang and T.-C. Wang, "Application of the grey LotkaVolterra model to forecast the diffusion and competition analysis of the TV and smartphone industries," Technological Forecasting and Social Change, vol. 106, pp. 37-44, 2016.

[26] T. Wei and T. Liu, "Evolution of high-value patents in reverse innovation: focus on Chinese local enterprises," Mathematical Problems in Engineering, vol. 2020, Article ID 8127096, 13 pages, 2020.

[27] C. Cordes and G. Schwesinger, "Technological diffusion and preference learning in the world of Homo sustinens: the challenges for politics," Ecological Economics, vol. 97, pp. 191-200, 2014.

[28] A. M. Ziegler, N. Brunner, and M. Kühleitner, "The markets of green cars of three countries: analysis using Lotka-volterra and Bertalanffy-Pütter models," Journal of Open Innovation: Technology, Market, and Complexity, vol. 6, no. 3, p. 67, 2020.

[29] A. S. Chakrabarti, "Stochastic Lotka-Volterra equations: a model of lagged diffusion of technology in an interconnected world," Physica A: Statistical Mechanics and Its Applications, vol. 442, pp. 214-223, 2016.

[30] M. L. Guilherme and J. F. Fontanari, "Influence of technological progress and renewability on the sustainability of ecosystem engineers populations," Mathematical Biosciences and Engineering, vol. 16, no. 5, pp. 3450-3464, 2019.

[31] L. C. M. Miranda and C. A. S. Lima, "Technology substitution and innovation adoption: the cases of imaging and mobile communication markets," Technological Forecasting and Social Change, vol. 80, no. 6, pp. 1179-1193, 2013.

[32] B. Meng, H. Kuang, E. Niu, J. Li, and Z. Li, "Research on the transformation path of the green intelligent port: outlining the perspective of the evolutionary game "Government-PortThird-Party organization"” Sustainability, vol. 12, no. 19, p. 8072, 2020.

[33] P. A. Geroski and M. Mazzucato, "Modelling the dynamics of industry populations," International Journal of Industrial Organization, vol. 19, no. 7, pp. 1003-1022, 2001.

[34] G. L. Zhang, A. Daniel, and V. M. Adams, "Technology evolution prediction using Lotka-Volterra equations," Journal of Mechanical Design, vol. 140, no. 6, pp. 61-101, 2018.

[35] T. Modis, "Technological forecasting at the stock market," Technological Forecasting and Social Change, vol. 62, no. 3, pp. 173-202, 1999.

[36] C.-T. Chang, C.-Y. Ku, and H.-P. Ho, "Fuzzy multi-choice goal programming for supplier selection," International Journal of Operations Research and Information Systems, vol. 1, no. 3, pp. 28-52, 2010. 
[37] A. H. I. Lee, H.-Y. Kang, C.-Y. Yang, and C.-Y. Lin, "An evaluation framework for product planning using FANP, QFD and multi-choice goal programming," International Journal of Production Research, vol. 48, no. 13, pp. 3977-3997, 2010.

[38] C.-T. Chang, "Multi-choice goal programming," Omega, vol. 35, no. 4, pp. 389-396, 2007.

[39] S.-Y. Wang, W.-M. Chen, and Y. Liu, "Collaborative product portfolio design based on the approach of multichoice goal programming," Mathematical Problems in Engineering, vol. 2021, Article ID 6678533, 16 pages, 2021.

[40] Z. Consulting, "2017-2022 China automotive lightweight market operation situation and investment strategy research report," 2016, https://www.chyxx.com/research/201610/ 459867.html.

[41] B. Zhang, P. Li, H. Zhou, and X. Yue, "China's auto industry upgrade process based on aging chain and coflow model," Complexity, vol. 2021, Article ID 3435953, 12 pages, 2021.

[42] Official website of China Automobile Industry Association, "Statistics of China automobile industry association," 2021, https://www.caam.org.cn/tjsj.

[43] L. Schrage, LINGO Release 8.0, LINDO System, Inc., Chicago, IL, USA, 2002.

[44] H.-C. Lee and C.-T. Chang, "Comparative analysis of MCDM methods for ranking renewable energy sources in Taiwan," Renewable and Sustainable Energy Reviews, vol. 92, pp. 883896, 2018.

[45] O. Mohsen and N. Fereshteh, "An extended VIKOR method based on entropy measure for the failure modes risk assessment-a case study of the geothermal power plant (GPP)," Safety Science, vol. 92, pp. 160-172, 2017.

[46] R. Shad, M. Khorrami, and M. Ghaemi, "Developing an Iranian green building assessment tool using decision making methods and geographical information system: case study in Mashhad city," Renewable and Sustainable Energy Reviews, vol. 67, pp. 324-340, 2017.

[47] A. Hafezalkotob and A. Hafezalkotob, "Extended MULTIMOORA method based on Shannon entropy weight for materials selection," Journal of Industrial Engineering International, vol. 12, no. 1, pp. 1-13, 2016.

[48] F. H. Lotfi and R. Fallahnejad, "Imprecise Shannon's entropy and multi attribute decision making," Entropy, vol. 12, no. 1, pp. 53-62, 2010.

[49] C.-L. Hwang and K. Yoon, Methods for Multiple Attribute Decision Making: Multiple Attribute Decision Making, Springer, New York, NY, USA, 1981.

[50] F.-D. Dong, W.-T. Li, and J.-B. Wang, "Propagation dynamics in a three-species competition model with nonlocal anisotropic dispersal," Nonlinear Analysis: Real World Applications, vol. 48, pp. 232-266, 2019.

[51] Q. Liu, S. Liu, and K.-Y. Lam, "Stacked invasion waves in a competition-diffusion model with three species," Journal of Differential Equations, vol. 271, pp. 665-718, 2021. 